\title{
Las relaciones entre América Latina y los Estados Unidos*
}

Agradezco al profesor Abraham Lowenthal por haber tomado la iniciativa de sugerir este intercambio de ideas entre personalidades de los Estados Unidos y de América Latina. El hecho de que asistan a esta reunión personas con trayectorias profesionales muy diferentes - gobierno, prensa, universidades o negocios- no sólo constituye una buena evidencia del creciente interés por el futuro de los asuntos interamericanos, sino también pone de relieve el alcance y la variedad de nuestras relaciones actuales.

Creo que este renovado interćs es extremadamente positivo y que debe estimular los esfuerzos hacia el mejoramiento de nuestras relaciones durante los próximos años. No hay duda que encuentros como ćstc juegan una parte importante en la profundización del diálogo entre los latinoamericanos y los norteamericanos. Ellos nos proporcionan oportunidades para comparar puntos de vista acerca de tópicos vitales de interés común, sin ningún otro compromiso que el de ser honestos unos con otros y de dedicarnos a la búsqueda de una comprensión mutua.

Es siempre fascinante, a la vez que un desafio político, revisar las tendencias y perspectivas de las relaciones entre América latina y los Estados Unidos. Obviamente, no puedo hablar en nombre de cada país latinoamericano ni tampoco en representación de América latina como un todo. Lo más que puedo hacer es expresar un punto de vista brasilero, en mi capacidad de Ministro de Relaciones Exteriores de un país que pertenece a la comunidad latinoamericana. Dada la diversidad de aspiraciones, opiniones y pasado histórico, que existe natura!mente entre los paíscs latinoamericanos, considero necesario hacer esta advertencia inicial. Para que mis palabras sean colocadas en su perspectiva correcta, añadiré que si bien una cuota de pronóstico es inherente al análisis diplomático de cualquier situación política, no es mi deseo parecer profético. Resistiré la tentación de predecir que sucederá tal o cual evento o combinación de circunstancias. Las ambigüedades del presente no lo garantizarían y mi propia posición de actor interesado en el drama político del hemisferio tampoco lo harian aconsejable.

Artículo $2^{\circ}$, Enrique lglesias. 
Constituye una coincidencia significativa el que nuestra reunión se efectúe bajo los auspicios de una institución académica que lleva e] nombre del Presidente Woodrow Wilson. Como fundador de la tradición liberal moderna en la política exterior norteamericana, el Presidente Wilson fue uno de los estadistas de nuestro siglo más dedicados a poner en línea la conducta internacional de todos los países (incluido el propio) con los preceptos éticos universales. Pero inclusive durante la administración del Presidente Wilson, los Estados Unidos enfrentaron problemas y conflictos con diversos países en América Latina. La política hemisférica del Presidente Wilson marcó una era y suscitó preguntas dilatadas, cuyas consecuencias aún conforman un capítulo inconcluso en nuestras relaciones.

La brecha entre los principios globales de las políticas del Presidente Wilson y su implementación en el hemisferio occidental ha sido tradicionalmente descrita como una paradoja. En realidad, esta descripción equivale a admitir que tal brecha no puede ser explicada racionalmente. Pero hoy en día, desde la perspectiva de más de medio siglo de historia, esta aparente paradoja ha comenzado, de hecho, a desaparecer. A medida que se desvanece, un tipo especial de orden complementario se hace aparente. En el largo plazo, y como una tendencia general, los temas interamericanos han ocupado tradicionalmente un segundo lugar en la política exterior global de los Estados Unidos. Bajo las demandas urgentes de sus intereses y responsabilidades globales, los Estados Unidos a veces transplantan en sus políticas latinoamericanas valores que no son necesariamente aplicables a la rcgión. Por lo tanto, la realidad latinoamericana a menudo es percibida a través de un filtro de ideas preconcebidas y, como una consecuencia, las políticas de los Estados Unidos tienden a estar distorsionadas. Después de un cuidadoso escrutinio, parece que los Estados Unidos tratan en realidad de duplicar en América Latina éxitos conseguidos en otros lugares o, más frecuentemente, buscar compensación por sus frustraciones en otras regiones. Pero los latinoamericanos no deberian quejarse por esta situación. Creo que la mejor forma de dar más peso a los temas latinoamericanos dentro de la política global norteamericana es tratar de eliminar la dependencia, sea ésta de orden cconómico o intelectuai.

Debo decir que las relaciones entre América Latina y los Estados Unidos cstán influenciadas por formas particulares de arcaísmo. Pasan alternativamente por fases "bucnas" y "malas", caracterizadas de acuerdo con el relativo "interés" o "desinterés", o bien por las "ganancias" o "pérdidas", experimentadados por Washington en conexión con los temas interamericanos. De hecho, nuestra relación ha estado basada en definiciones unilaterales, en las que América Latina ha sido contemplada más como un objeto de politicas que como un socio en un diálogo abierto y franco. 
Por to menos en lo que respecta a los países latinoamericanos, este enfoque está claramente agotado. Por esa misma razón, nuestras relaciones están hoy marcadas por reclamos crecientes y no siempre bien comprendidos para que se efectúen revisiones o cambios en ellas. Cualquier interpretación de la historia de este siglo no deja lugar a dudas acerca del constante interés de América Latina por cambiar el tenor de sus relaciones con los Estados Unidos. Por su persistencia y volumen, estas demandas ayudaron a generar un reconocimiento de que la estructura de nuestras relaciones es obsoleta y clama por un cambio urgente.

Para ser auténtico, nuestro diálogo debe reflejar nuestras diferentes visiones del mundo. No debería esperarse que los latinoamericanos y los norteamericanos definieran los grandes problemas contemporáneos en la misma forma, o establecieran las mismas escalas de prioridades para sus soluciones. La solidaridad recíproca y los intereses compartidos deberían permitirnos explorar las áreas donde nuestros intereses convergen o se sobreponen y eliminar fricciones innecesarias, o al menos limitarlas a zonas de inevitable conflicto. Confio en que el respeto mutuo y la confianza prevalecerán siempre y que trataremos de negociar nuestras diferencias con buena fe. No debería haber lugar para imposiciones, incluso si son bien intencionadas, entre países amigos.

Si bien nuestros problemas políticos $y$ económicos son tan diversos como nuestros respectivos mundos culturales, en este hemisferio compartimos un tipo de vida inspirado por las premisas y aspiraciones de la civilización occidental. La comprensión que existe entre nosotros debe ser nutrida por los valores fundamentales de esta herencia común.

Puesto que nuestro diálogo está influido por este pasado ético y por los intereses y la amistad que surgen de él, aquel tiene lugar en circunstancias materiales que son bastante disparejas para cada uno de los que dialogan. Los Estados Unidos son uno de los miembros más importantes del mundo industrializado, mientras que América Latina es parte del Tercer Mundo. Males que son un insulto a la dignidad humana, tales como la pobreza, la enfermedad, el hambre y la ignorancia, constituyen una realidad más cercana a América Latina que a los Estados Unidos.

Todas las energías materiales y espirituales que América Latina puede generar están puestas al servicio de su lucha por el desarrollo. El significado y la tremenda urgencia de nuestra lucha, sin embargo, nọ es siempre evaluada en forma correcta, aún por aquéllos países desarrollados que comparten con nosotros tantos valores y aspiraciones importantes. Sería deseable que tales países, incluido los Estados Unidos, adoptaran actitudes más positivas, capaces de facilitar la remoción de los obstáculos internacionales al desarrollo, acelerando así los esfuerzos de América Latina para desarrollarse, en conformidad con los ideales de la civilización occidental. 
En años recientes, las relaciones entre América Latina y los Estados Unidos prácticamente se han estancado. Programas de ayuda paternalista fueron reemplazados por la indiferencia, que se vio mitigada sólo en muy contados casos. Ultimamente, es verdad que la apatía dio paso a un gran activismo, pero esto no ha hecho nuestras relaciones tan dinámicas como deberían ser, considerando la naturaleza y la seriedad de los problemas que enfrent an los pueblos de este hemisferio.

Creo que las relaciones interamericanas han perdido su especificidad y se puede decir que van a la deriva. Sus aspectos principales se identifican con unos cuantos problemas globables, los cuales, en verdad, van más allá de la región. Los slogans que disimulan la falta de una política consensual ya no pueden ser usados, a medida que desaparecen, se revela un vacio de ideas políticas. A veces, estoy inclinado a preguntarme si esta situación - -en vista de sucesos pasados- es enteramente insatisfactoria. Desde el punto de vista del futuro, sin embargo, no creo que en este hemisferio podamos permitirnos el no ejercitar nuestra opción básica, que es la de trabajar todos juntos para conseguir que nuestras relaciones procedan sobre bases normales, esto es, de acuerdo con una línea de cooperación.

Dentro del contexto mínimo en que nos hallamos, han encontrado apoyo dos recomendaciones: una que sostiene que América Latina no debería seguir siendo tratada como un bloque o una unidad única puesto que, como podría parecer bastante obvio, cada país tiene valor per se y una individualidad que no debemos menospreciar, y una segunda opción que realmente ha estado en forma embriónica desde la era de la Alianza para el Progreso, que implica la división de los países latínoamericanos en dos grupos, y cuyo elemento diferenciador es el grado de alineamiento de sus prácticas políticas con las actuales prioridades de Washington.

Sin embargo, la sola aplicación de estas fórmulas está lejos de agotar las opciones de las relaciones interamericanas. América Latina, de hecho, es proclive a las simplificaciones. Las relaciones en el hemisferio han alcanzado un punto tan bajo que queda librado a su curso natural el que se deterioren hasta llegar a algún tipo de conflicto entre nuestros objetivos $y$ métodos de acción. Deberíamos, por lo tanto, descubrir nuevas formas de organizar nuestras relaciones. De otro modo, nos será difícil mantener una relación armoniosa en el futuro.

Ultimamente han habido unas cuantas fallas. Me atrevo a decir que la causa de ello radica tanto en las etiquetas que se han puesto a las politicas como en sus esencias y sustancias. Jamás podremos convertir nuestros anhelos de cambio en una realidad operativa. Los antiguos vicios de nuestras relaciones resisten el asedio del tiempo. Y sin embargo, estoy convencido de que, después de tantas dificultades, ha llegado el momento de cambiar el enfoque.

Deberíamos, por supuesto, poseer más confianza en nuestra habilidad mutua para comunicarnos. Las relaciones interamericanas no 
tendrían que reducirse a simpies modelos de acción y reacción, puesto que constituyen un proceso de permanente intercambio de ideas $y$, por ello, un ejercicio de persuación mutua. Cada diálogo presupone el bilateralismo, esto es, la admisión de que podemos sostener diferentes opiniones $y$ de que podemos trabajar unidos para reconciliarlas. Debería comprenderse que los países latinoamericanos, como sociedades, están sufriendo cambios muy rápidos y que no puedén estar expuestos a reacciones superficiales derivadas de un largo período de dependencia económica, intelectual y moral. Todos los grupos deben hacer oír su voz a través de sus representantes autorizados. Ha terminado la era de las actitudes y decisiones unilaterales. Esta aproximación aparentemente simple podría implicar una revolución en la forma de manejar asuntos interamericanos.

Creo que la experiencia del Brasil dentro de Sudamérica puede ser útil para la consideración del estado de las relaciones interamericanas como un todo. Nosotros los brasileros tenemos diez vecinos inmediatos en nuestro continente, cada uno de ellos comprimido contra los otros dentro de un área delimitada por dos océanos. Inclusive los dos países sudamericanos que no tienen limites con nosotros se encuentran muy cercanos. A primera vista, estas circunstancias parecen tener un significado únicamente geográfico, pero son extremadamente relevantes para la diplomacia brasilera, puesto que nos han enseñado a aceptar y a apreciar la diversidad de nuestros países vecinos, como también a respetar sus puntos de vista. Nuestra política dentro de América Latina está informada permanentemente por la realidad de esa relación de vecindad, una realidad compleja, puesto que no se duplica en el tiempo o en el espacio. Esta vecindad nos ha confrontado con problemas concretos e inmediatos que a causa de su naturaleza y variedad no pueden ser reducidos a ábstracciones temáticas. Esta actividad diplomática es llevada a cabo enteramente bajo la égida de un profundo compromiso de solidaridad, basado en la premisa de que los acuerdos y los entendimientos internacionales derivan su legitimidad y eficacia del grado de satisfacción que confieren a todos los grupos comprometidos en ellos.

Un ejemplo concreto de la aplicación práctica de esta doctrina, que esperamos adquiera creciente importancia en nuestro hemisferio, fue la firma del Tratado de Cooperación en la Región Amazónica, que fue firmado en Brasilia hace poco más de dos meses. Este instrumento legal, que reúne a los ocho países amazónicos, constituye un signo de esperanza para un área que, a pesar de estar situada en el corazón de Sudamérica, nunca antes ha tenido un marco internacional adecuado. En términos diplomáticos, el tratado también cierra una fase exploratoria. Junto con otros instrumentos regionales que están actuando, ella anuncia en forma dramática el establecimiento de nuevas formas de relaciones internacionales dentro de América Latina. Por lo tanto, el Tratado Amazónico es válido como tal, y también por aquello que representa para el futuro de las relaciones intraregionales en América Latina, 
así como para la coexistencia de los países latinoamericanos con el resto del mundo.

Como ya lo he mencionado, en América Latina se está produciendo hoy un proceso acelerado de cambio. En los diferentes países de la región, han ido evolucionando las mentalidades colectivas, $y$ hoy en día existe la convicción general de que este espiritu de cambio también debe alcanzar a las relaciones interamericanas. Estamos viviendo un período de transición en nuestras relaciones, pero sólo será posible inyectar vida real en ellas si estamos claramente determinados a enfrentar las nuevas realidades hemisféricas en forma colectiva.

Es un hecho el que nuestras relaciones durante este siglo hayan estado basadas en la participación relativamente restringida de América Latina en temas de interés para el mundo en general, como también en una asimetría fundamental entre los principales poderes industriales y un número razonable de países en desarrollo. La persistencia de esta estructura dio origen a racionalizaciones recurrentes $\mathrm{e}$ incluso a pautas hegemónicas de conducta.

Hoy prevalecen condiciones diferentes en el mundo. El sistema colonial, que fue un elemento significativo dentro de la vida internacional, se tornó obsoleto y sus mecanismos, inservibles. Vivimos en un mundo multipolar. Todos los estados tienen nuevas oportunidades frente a ellos, sin relación con sus tamaños. En América Latina, a medida que comenzamos a cosechar los primeros frutos del desarrollo, los países se vuelven capaces de participar más activamente en la vida internacional. Nuestro presunto aislamiento se ha desvanecido, en tanto que el desequilibrio de poder existente ya no basta para definir el proceso político, ni en nuestra región ni en el resto del mundo.

Es importante reconocer que el predominio del eje Norte-Sur en las relaciones interamericanas será balanceado gradualmente por otros impulsos. Los lazos que unen a los países latinoamericanos están haciéndose cada vez más fuertes; el Tratado Amazónico es un ejemplo de lo que puede conseguirse. Otras posibilidades interesantes son igualmente dignas de exploración, tales como el establecimiento de vías concretas, institucionalizadas bilateral y multilateralmente, para hacer que los países caribeños, que son tan latinoamericanos como el Brasil, se unan más estrechamente con el resto de América Latina.

En la misma línea, una postura ecuménica ha agrandado la gama de opciones disponibles para las políticas exteriores del Brasil y de otros países latinoamericanos. Después de un largo periodo de aislamiento, América Latina está retornando una vez más a su vocación universal y cada día aumentará su interés por encontrar nuevas formas de cooperación con paises de todo el mundo. Brasil está buscando esa meta, al estrechar sus lazos con naciones industrializadas, tales como la República Federal de Alemania, Francia, Gran Bretaña y Japón; al aumentar su comercio con países del Tercer Mundo, tales como Ni- 
geria, y con estados de Europa Oriental, tales como Polonia y la Repúbiica Democrática Alemana; al establecer relaciones diplomáticas con la República Popular China; y al reconocer con prontitud la independencia de Angola, Mozambique y otras ex colonias de lengua portuguesa.

En el nivel interamericano multilateral, deberíamos estar de acuerdo en consagrar nucvamente la Organización de Estados Americanos a los principios de su carta consultiva, principios que nacieron de una larga, y no siempre pacífica, relación en este hemisferio. Estos principios constituyen la mejor garantía a disposición de todos los estados que pertenecen al sistema interamericano. Y para cada estado individual, ellos acarrean compromisos legales, morales y políticos, que por su naturaleza no admiten calificaciones, restricciones o distinciones selectivas.

Estos principios deben ser observados durante el actual período de transición hacia una relación más balanceada y equitativa. Me refiero especialmente a la obligación de actuar de buena fe y de respetar las normas del Derecho Internacional. Me refiero también a los principios de no intervención, independencia, soberanía e igualdad de los estados y de respeto por los derechos humanos. Es sobre la base de estos preceptos que la oEA debe ser reorientada a fin de poder ejercitar sus funciones como el principal foro negociador para la cooperación interamericana, reformando para este fin su maquinaria económica, de manera que pueda contribuir efectivamente al desarrolio libre y sin trabas de sus estados miembros. En esta área, como en otras, los Estados Unidos deben unirse a nuestro esfuerzo cooperativo y proyectar un perfil menos ortodoxo y más generoso que el que mostró en la última sesión de la Asamblea General de la OEA en junio de 1978.

América Latina está básicamente unida en lo referente a dos preguntas centrales: soberanía y desarrollo. Como ex colonias luchando por obtener el progreso social y económico, los diferentes países latinoamericanos son particularmente sensitivos a los problemas que se plantean en estas dos áreas básicas.

Existe una tradición de interferencia en América Latina. A pesar de su éxito ocasional, la interferencia es difícilmente una opción que puede ser mantenida en el largo plazo, puesto que crea su propia oposición. Es dudoso que se pueda obtener algo duradero y constructivo de la aplicación simultánea de presiones liberales en los niveles políticos y conservadores dentro del campo cconómico. Creo que, básicamente, ningún país en nuestro hemisferio, o en cualquier otro lugar, está en condiciones de juzgar unilateralmente a otros países. Tampoco creo que puedan ser efectivas en alguna forma las recriminaciones o interferencias. El diálogo entre naciones amigas no deberia estar jamás contaminado por un clima de guerra fría, con el riesgo de cometer una gran injusticia y de causar fricciones en forma inevitable.

$E_{n}$ este contexto general, me imagino que ustedes quisieran tal vez conocer mis puntos de vista en lo referente al importante tema de los 
derechos humanos y su peso en las relaciones futuras entre los países latinoamericanos y los Estados Unidos.

Este no es el momento para reformular la posición tomada por el gobierno del Brasil en relación con la consideración internacional de los derechos humanos, pero a manera de introducción, me gustaría recalcar que nuestra posición radica en tres conceptos básicos: 1) la obligación del Estado de garantizar el respeto por los derechos humanos dentro de sus fronteras nacionales, y de castigar cualquier violación de estos derechos de acuérdo con sus leyes nacionales; 2) la inadmisibilidad de la interferencia extranjera en materias referentes a leyes nacionales y 3) el apoyo a la cooperación internacional para la afirmación de los derechos humanos de naturaleza política, económica y social.

La referencia a estos tres conceptos básicos da una idea de los diversos aspectos que presenta el tema de los derechos humanos. Aceptar la complejidad de este asunto es el primer paso para tratarlo en una forma serena, racional y constructiva. Es verdad que las realidades que enfrentamos son duras e intratables, pero ésta debe ser una razón adicional para que hagamos un esfuerzo realmente objetivo en el campo de la cooperación internacional para afirmar los derechos humanos en todos sus aspectos.

Para poder avanzar en esta delicada área, sería conveniente corregir ciertas percepciones que todavía estorban nuestro diálogo. Por ejemplo, permanece la convicción de que el individuo debería ser considerado en abstracto, como si existiese fuera de la sociedad en que vive. Como resultado de ello, es costumbre tratar el tema de los derechos humanos en una forma igualmente abstracta, sin tomar en consideración las condiciones sociales y económicas y las vicisitudes históricas que nos hace diferentes como países y como personas. Para que tenga sentido práctico, cualquier análisis de los problemas relacionados con los derechos humanos debería ser comprensivo en su enfoque e incorporar otros datos.

Por otra parte, muchas de las declaraciones hechas sobre este tema por lo menos implican el punto de vista de que, en los países en desarrollo, puede hacerse una distinción en lo referente al valor que asigna a los derechos humanos en los campos político, económico y sociales, enfatizando el primero, es decir, el de los derechos políticos, en detrimento de los otros. En el nivel internacional, se da un redoblado énfasis a los derechos políticos, cuya observancia universal no entrañaría ninguna responsabilidad para los países desarrollados. Sin embargo, no se divisan nuevos compromisos en lo referente a la creación de condiciones para el desarrollo que a su vez permitirían a toda la humanidad ejercer plenamente sus derechos económicos - esenciales, una meta que obviamente depende de las concesiones concretas que efectúe el mundo industrializado.

No deseo enfatizar aquí otros aspectos bien conocidos de este tema. Al recalcar ciertas situaciones, y olvidar otras, de acuerdo con matices 
estratégicos o ideológicos, puede aparecer una discrepancia evidente entre la conveniencia política y la consistencia ética. En conexión con esto, no debe olvidarse la posibilidad de que un país frustre los propósitos de un instrumento al cual ha adherido poniendo múltiples reservas a su implementación práctica.

Los comentarios que acabo de hacer están dirigidos exclusivamente a la forma en la cual el tema de los derechos humanos ha sido presentado internacionalmente. En cuanto a su sustancia, estoy convencido de que existe un concenso en este hemisferio sobre la absoluta necesidad de respetar los derechos humanos. Por lo tanto, ningún país puede considerarse a sí mismo único en su dedicación a la causa de los derechos humanos. Todos nosotros, tanto los latinoamericanos como los norteamericanos, tenemos una larga tradición de lucha por la plena afirmación de estos derechos en todas sus formas. El tema de la cooperación internacional para la afirmación de los derechos humanos estará ciertamente en la agenda del hemisferio durante los años venideros. La consideración futura de este tema deberia estar libre de presunciones pasadas de moda y de las percepciones erróneas actuales.

Hay otro punto fundamental que permanecerá en nuestra agenda: aquel que se refiere al rol del comercio internacional como un factor en el desarrollo económico. En este campo se han alcanzado pocos progresos, o ninguno, a pesar del interés primario que en él tiene toda América Latina.

A pesar de todos los esfuerzos negociadores efectuados por los países en desarrollo, especialmente por los latinoamericanos, no se han encontrado soluciones a los problemas que nos han estado molestando por décadas, tales como la inestabilidad tradicional de los precios internacionales de los productos exportados por los países en desarrollo. Si hay algo nuevo en el campo de las relaciones económicas internacionales, es la imposición de nuevas barreras al comercio entre el norte y el sur. Me refiero tanto al proteccionismo comercial como al creciente número de obstáculos a la transferencia internacional de tecnología. Estoy convencido de que estos dos temas presentan desafios fundamentales y que ya es tiempo para que discutamos la positiva contribución que el Norte debería hacer a los paises del Sur en sus esfuerzos para desarrollarse.

En este punto, me gustaría referirme brevemente a un tema que tiene un alcance más amplio, pero que incide directamente en los asuntos que ahora estamos considerando. Creo que la reunión económica cumbre celebrada recientemente en Bonn entrc los siete países más desarrollados del mundo merece nuestra especial atención. Limitaré mis comentarios al interés demostrado por los países occidentales en Bonn para la creación de una categoría especial de países en desarrollo, denominados los países en desarrollo avanzados (asi llamados en inglés ADC's). Tales paises no tendrian derecho a los beneficios de 
un tratamiento más favorable, que eventualmente podría ser concedido a los países en desarrolio.

Desde nuestro punto de vista, es inquietante que se haya expresado tal idea por parte de los paises occidentales. Brasil, así como-creo yo- otros países latinoamericanos que se encuentran en una etapa similar de desarrollo, se propone cumplir sus compromisos internacionales. No hay una razón económica válida para que estos países sufran una discriminación que vaya a afectar en forma adversa el ritmo de su desarrollo económico. No se nos puede culpar por las actuales deficiencias de la economía mundial y sería injustificable aumentar las cargas propias del subdesarrollo, que pesan actualmente sobre nosotros, añadiendo nuevos mecanismos capaces de segregarnos 0 , al menos, de limitar nuestra participación en los resultados positivos que pueden surgir de las negociaciones Norte-Sur.

Es sorprendente que el pensamiento desarrollado en Bonn haya adoptado esta orientación. Habría sido mucho más positivo haberse concentrado en nuevas formas para incluir a los países en desarrollo en el proceso internacional de toma de decisiones. Parece que en la opinión de las naciones industrializadas, a pesar del nivel de desenvolvimiento alcanzado por algunos países en desarrollo, éstos no están en cierto modo autorizados para participar equitativamente en las decisiones encaminadas a establecer pautas para el funcionamiento de la economía internacional. Por el contrario, se esgrime el hecho de haber alcanzado tal nivel de desarrollo como una razón para la imposición de nuevas cargas sobre sus economías. De seguirse esta senda, ella no conduciría a una interacción más productiva entre los países ricos y los pobres.

Más aún, es difícil no establecer una conexión entre esta nueva idea y la circunstancia de que algunas importantes negociaciones comerciales que se están efectuando ahora entre países en desarrollo y desarrollados han entrado recién en su etapa decisiva. Temo que los países industrializados en Bonn hayan tenido la tentación de jugar a la política con esta delicada situación. Las naciones en desarrollo ya se han dividido, con la emergencia del concepto de "paises menos desarrollados", y se han dividido nuevamente por el encumbramiento de los países exportadores de petróleo. Ciertamente podemos arreglarnos sin divisiones adicionales.

En esta luz, alabaremos cualquier esfuerzo por parte de los países industrializados para disipar estas preocupaciones. Una forma de hacerlo sería que las siete naciones altamente desarrolladas establecieran compromisos unilaterales más poderosos que los de los otros miembros de su grupo, en el campo del comercio y la cooperación para el desarrolio de los "países menos desarrollados"

Espero que esta apreciación de las relaciones entre América Latina y los Estados Unidos resulte útil para fomentar el entendimiento mutuo. 
No creo en recriminaciones, ni creo que las políticas puedan ser reducidas a un ejercicio cartesiano. Estoy convencido de que la sola reproducción de viejos moldes no es suficiente para desarrollar nuestras relaciones en forma adecuada. Inspirados por nuestras creencias y por nuestro pasado común, deberíamos intentar el establecimiento de una serie de entendimientos operativos, capaces de responder a las necesidades básicas de cada momento, y preparar el camino para efectuar cambios cualitativos en nuestras relaciones. Los slogans pasaron de moda en nuestro hemisferio. Pero no ha disminuido la necesidad de aguzar la perspicacia política. No habría justificación para permitir que nuestras relaciones tradicionales naufragaran hasta alcanzar el nivel de un capítulo secundario en la política internacional. Por el contrario, como he tratado de demostrar, estoy seguro de que si mantenemos una capacidad de diálogo racional, y si manejamos en una forma equilibrada todos los temas concernientes a este hemisferio, seremos capaces de transformar nuestras relaciones y colocarlas firmemente sobre una base más productiva y mutuamente satisfactoria. Las tradiciones, realidades $y$ aspiraciones de las Américas garantizan la fe en nuestra capacidad para trabajar unidos para lograr nuestro objetivo común y de esta manera, enfrentar en forma madura y solidaria los temas de la próxima década. Hablando por Brasil, debo manifestar claramente que nuestra posición es positiva y que estamos listos para seguir el camino del diálogo y la negociación. 